\title{
State Space Solution for a Pile Subjected to Combined Lateral and Vertical Loads
}

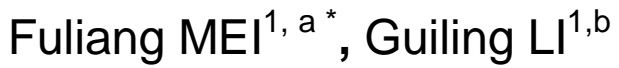 \\ ${ }^{1}$ Institute of Mechanics, Jiaxing University, Jiaxing, China \\ âFImei2005@126.com, bLgljx@126.com
}

\begin{abstract}
Keywords: Soil-structure interaction, pile, lateral and vertical loads, state space method, sub-grade reaction model, three parameter model

Abstract. A state space solution for a partially embedded pile under combined lateral and vertical loads is developed. Two sets of state space equations above and below ground are deduced from Euler-Bernoulli's and Winkler's linear elastic foundation beam theories respectively, in which the coefficient of sub-grade reaction is defined by a three parameter model. Two pile shafts above and below ground are divided into uniform sub-sections by means of sub-section approximation method in order to make the above nonlinear state space equations become linear. The expressions of bending deformations and internal forces for all the sub-sections are deduced by virtue of matrix theory, boundary conditions, continuity-smooth conditions at the interface between two adjacent sub-sections. Computational results for a specific pile under specified lateral and vertical loads reveal that the present solution agrees well with existing semi-analytical one, and has higher convergence and accuracy.
\end{abstract}

\section{Introduction}

In many a circumstance, a number of piles are used to be subjected to combined lateral and vertical loads. In consequence, the prediction of responses for a single pile under lateral and vertical loads simultaneously is urgently an important event in the design, construction and application of pile foundation.

Based on a sub-grade reaction method, a wealth of solutions for a single pile subjected to combined lateral and vertical loads include: finite element ones (Karthineyan et al.,2006; Karthigeyan et al.,2007; Zheng et al.,2009), power series ones (Zhao et al.,1987; Wang et al.,2005), and variation principle ones (Han et al.,2000). In the aforementioned solutions, the coefficient of sub-grade reaction was assumed to remain constant or linearly increase with depth (Wang et al., 2005), while it was equal to be zero in the vicinity of ground, so that there is a certain difference between these results and practical ones. In a few decades, a three parameter model, which can authentically represent the distribution of sub-grade reaction with depth and lateral deformation-resisting capability of soil in the vicinity of ground, was presented for predicting the responses of a pile subjected to a lateral load in literature (H.L.Wu et al.,2000; B.H.Wang et al.,1999; H.Matlock et al.,1960). Zhang L. et al. (2011) obtained the semi-analytical solution of responses for a pile subjected to oblique loads by the three parameter model in H.L.Wu et al.(2000).

In this treatise a state space method to solve internal forces and deformations for a partially embedded pile under vertical and lateral loads is put forward. State space equations for the pile shafts above and below ground are given according to Euler-Bernoulli's and Winkler' linear elastic beam theories and sub-grade reaction model with a three parameters, and also state space equations for the sub-sections above and below ground by virtue of sub-section approximation approach are provided. The expressions of internal forces and deformations for all the sub-sections are deduced from matrix theory, boundary conditions and continuity-smooth conditions at the interface between two adjacent sub-sections. Finally the present solution for a specific pile under a specified oblique load is compared with existing semi-analytical one. 


\section{State Space Solution}

Consider a uniform cross-section and partially embedded pile subjected to a combined lateral and vertical load as shown in Fig.(1), here $N_{P}, Q_{p}$ and $M_{p}$ are vertical load, horizontal load and moment load on the pile head respectively . $H_{0}$ and $H_{1}$ are the pile lengths above and below ground respectively.

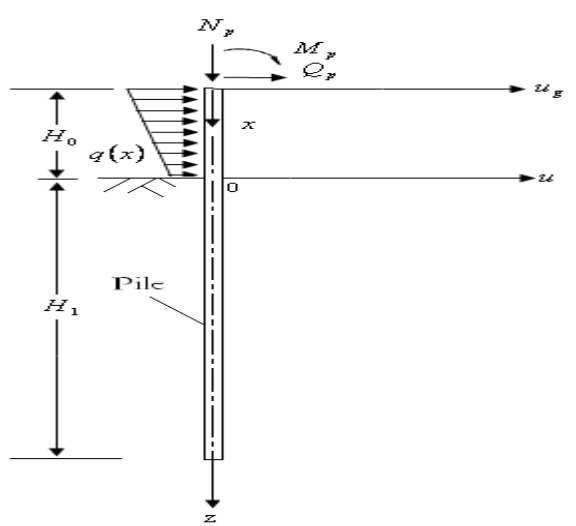

Figure.1 Schematic diagram of a pile under lateral and vertical loads

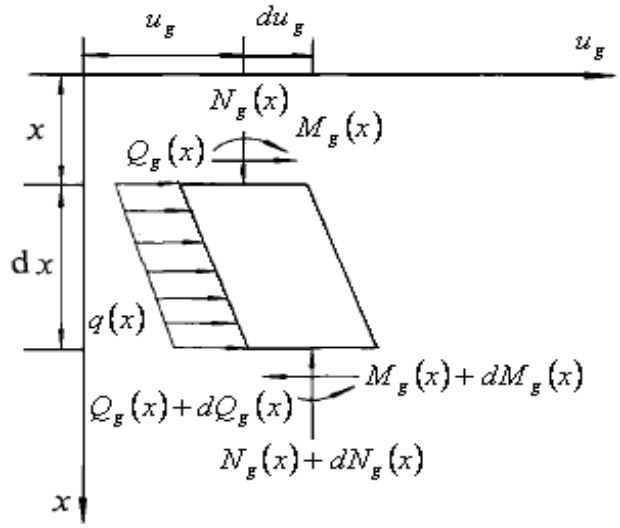

Figure. 2 force analysis of a pile shaft above ground

The force analysis and a local coordinate system for the pile shaft above ground are illustrated in Figure.(2) in which $u_{g}(x), \varphi_{g}(x), M_{g}(x), Q_{g}(x)$ and $N_{g}(x)$ stand for horizontal displacement, declination, moment, shear force and axial force at a depth $x$, respectively.

We can deduce a set state space equations above ground from Euler-Bernoulli's beam theory as

$$
\frac{d}{d x}\left\{\begin{array}{l}
u_{g}(x) \\
M_{g}(x) \\
Q_{g}(x) \\
\varphi_{g}(x)
\end{array}\right\}=\left[\begin{array}{cccc}
0 & 0 & 0 & 1 \\
0 & 0 & 1 & -N_{g}(x) \\
0 & 0 & 0 & 0 \\
0 & 1 / E I & 0 & 0
\end{array}\right]\left\{\begin{array}{l}
u_{g}(x) \\
M_{g}(x) \\
Q_{g}(x) \\
\varphi_{g}(x)
\end{array}\right\}+\left\{\begin{array}{l}
0 \\
0 \\
q(x) \\
0
\end{array}\right\}
$$

Where $E I$ is the pile flexural stiffness, $N_{g}(x)=N_{P}+f_{0} x$ in which $f_{0}$ denotes the growth factor of axial force resulting from self-weight, and $q(x)=q_{0}+\Delta q x / H_{0}$ in which $q_{0}, q_{0}+\Delta q$ are the distributed loads at the pile head and ground respectively. It is noted that Eq.(1)is a set of non-homogeneous differential equations of coordinate $x$ with variable coefficients .

Similarly, the force analysis and a local coordinate system for the pile shaft below ground are depicted in Fig.(3), in which $u(z), \varphi(z), M(z), Q(z)$ and $N(z)$ are horizontal displacement, rotation angle, moment, shear force and axial force at a depth $z$, respectively.

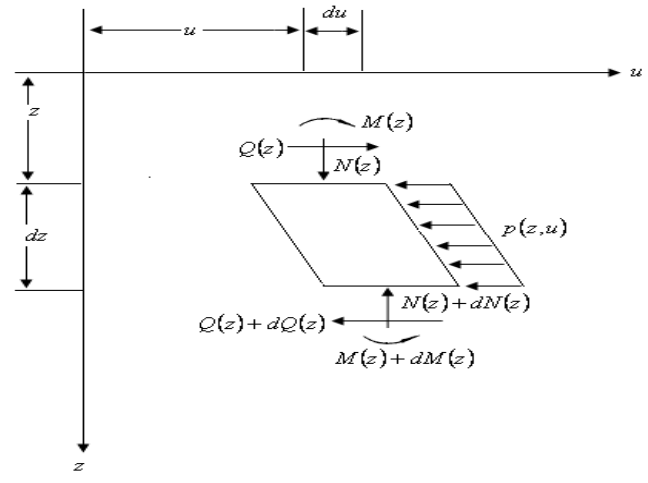

Figure. 3 force analysis of a pile shaft below ground

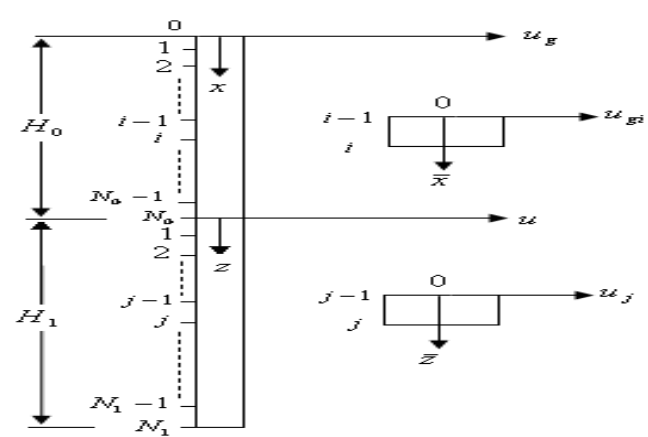

Figure. 4 schematic diagrams of sub-sections and local coordinate systems 
We can obtain another set of state space equations below ground according to Winker's linear elastic foundation beam theory as:

$$
\frac{d}{d z}\left\{\begin{array}{l}
u(z) \\
M(z) \\
Q(z) \\
\varphi(z)
\end{array}\right\}=\left[\begin{array}{cccc}
0 & 0 & 0 & 1 \\
0 & 0 & 1 & -N(z) \\
-k(z) b & 0 & 0 & 0 \\
0 & 1 / E I & 0 & 0
\end{array}\right]\left\{\begin{array}{l}
u(z) \\
M(z) \\
Q(z) \\
\varphi(z)
\end{array}\right\}
$$

Where $N(z)=N_{p}+f_{0} H_{0}+f_{1} z$ in which $f_{1}$ also is the growth factor of axial force due to combined self-weight and lateral friction of the pile, say, $f_{1}=\gamma_{c} A_{s}-\mu \tau / 2$ here $\gamma_{c}, A_{s}, \mu$ and $\tau$ stand for the specific gravity under water, cross-section area, pile perimeter, limit friction[4],respectively; the coefficient of sub-grade reaction $k(z)$ is calculated by H.L.Wu et al. (2000) as $k(z)=m\left(z_{0}+z\right)^{n}$ in which $z_{0}$ is the equivalent depth standing for the lateral deformation-resisting capability of soil at the ground, $m$ the proportionality coefficient of soil, $n$ the resistance index of soil. It also is noticed that Eq.(2) is a set of homogeneous differential equations of coordinate $\mathrm{z}$ with variable coefficients.

When the pile head is free, the state space variables ( $a t x=0)$ is given by

$$
\left[\begin{array}{llll}
u_{g}(0) & M_{g}(0) & Q_{g}(0) & \varphi_{g}(0)
\end{array}\right]^{T}=\left[\begin{array}{llll}
u_{g}(0) & M_{p} & Q_{p} & \varphi_{g}(0)
\end{array}\right]^{T}
$$

Where $u_{g}(0)$ and $\varphi_{g}(0)$ are the horizontal displacement and rotation angle to be determined.

When the pile base is free, the state space variables (at $z=H_{1}$ ) is given by

$$
\left[u\left(H_{1}\right) \quad M\left(H_{1}\right) \quad Q\left(H_{1}\right) \quad \varphi\left(H_{1}\right)\right]^{T}=\left[\begin{array}{llll}
u\left(H_{1}\right) & 0 & 0 & \varphi\left(H_{1}\right)
\end{array}\right]^{T}
$$

Here $u\left(H_{1}\right)$ and $\varphi\left(H_{1}\right)$ are the horizontal displacement and rotation angle to be determined.

When the pile base is fixed, the state space variables (at $\left.z=H_{1}\right)$ is expressed by

$$
\left[\begin{array}{llll}
u\left(H_{1}\right) & M\left(H_{1}\right) & Q\left(H_{1}\right) & \varphi\left(H_{1}\right)
\end{array}\right]^{T}=\left[\begin{array}{llll}
0 & M\left(H_{1}\right) & Q\left(H_{1}\right) & 0
\end{array}\right]^{T}
$$

Here $M\left(H_{1}\right)$ and $Q\left(H_{1}\right)$ are the bending moment and shear force to be determined.

Continuity-smooth conditions at the ground can be represented as

$$
\left[\begin{array}{llll}
u_{g}\left(H_{0}\right) & M_{g}\left(H_{0}\right) & Q_{g}\left(H_{0}\right) & \varphi_{g}\left(H_{0}\right)
\end{array}\right]^{T}=\left[\begin{array}{llll}
u(0) & M(0) & Q(0) & \varphi(0)
\end{array}\right]^{T}
$$

It is noted that the exact solutions for Eqs.(1) and (2) are directly difficult to solve. Thus we have to use a numerical method. To avoid difficulties in solving directly Eqs.(1) and (2) with variable coefficients, two pile shafts above and below ground are divided into $N_{0}$ and $N_{1}$ uniform sub-sections according to a sub-section approximation approach, here two sub-section lengths above and below the ground are $\Delta x=H_{0} / N_{0}$ and $\Delta z=H_{1} / N_{1}$ respectively. And then two local coordinate systems for a sub-section above and below ground are depicted in Fig.(4) respectively.

When $N_{0}$ is large enough, the axial force $N_{g i}$ and the horizontally distributed load $q_{i}$ for the $i$ th sub-section above the ground are assumed to be constant, and they are calculated as: $N_{g i}=N_{p}+f_{0}(2 i-1) \Delta x / 2$ and $q_{i}=q_{0}+\Delta q(2 i-1) \Delta x /\left(2 H_{0}\right)\left(i=1,2, \mathrm{~L}, N_{0}\right)$ respectively. And a set of state space equations for the $i$ th sub-section above ground are 


$$
\frac{d}{d \bar{x}}\left\{\begin{array}{l}
u_{g i}(\bar{x}) \\
M_{g i}(\bar{x}) \\
Q_{g i}(\bar{x}) \\
\phi_{g i}(\bar{x})
\end{array}\right\}=\left[\begin{array}{cccc}
0 & 0 & 0 & 1 \\
0 & 0 & 1 & -N_{g i} \\
0 & 0 & 0 & 0 \\
0 & 1 / E I & 0 & 0
\end{array}\right]\left\{\begin{array}{l}
u_{g i}(\bar{x}) \\
M_{g i}(\bar{x}) \\
Q_{g i}(\bar{x}) \\
\varphi_{g i}(\bar{x})
\end{array}\right\}+\left\{\begin{array}{l}
0 \\
0 \\
q_{i} \\
0
\end{array}\right\} \quad\left(i=1,2, \mathrm{~L}, N_{0}\right)
$$

Where $u_{g i}(\bar{x}), M_{g i}(\bar{x}), Q_{g i}(\bar{x})$ and $\phi_{g i}(\bar{x})$ are the horizontal displacement, the bending moment, shear force and rotation angle at a depth $\bar{x}$ respectively.

$$
\text { Let } \mathbf{H}_{g i}=\left[\begin{array}{cccc}
0 & 0 & 0 & 1 \\
0 & 0 & 1 & -N_{g i} \\
0 & 0 & 0 & 0 \\
0 & 1 / E I & 0 & 0
\end{array}\right] \quad \mathbf{B}_{g i}=\left\{\begin{array}{l}
0 \\
0 \\
q_{i} \\
0
\end{array}\right\}
$$

Based on matrix theory, we can obtain a set of relationship equations between two state space variables at the top and bottom of the $i$ th sub-section above ground

$$
\mathbf{U}_{g i}(\Delta x)=\mathbf{T}_{g i} \mathbf{U}_{g i}(0)+\mathbf{A}_{g i}
$$

Where $\mathbf{U}_{g i}(\Delta x)=\left[\begin{array}{lllll}u_{g i}(\Delta x) & M_{g i}(\Delta x) & Q_{g i}(\Delta x) & \varphi_{g i}(\Delta x)\end{array}\right]^{T}$ and $\mathbf{U}_{g i}(0)=\left[\begin{array}{llll}u_{g i}(0) & M_{g i}(0) & Q_{g i}(0) & \varphi_{g i}(0)\end{array}\right]^{T}$ are the state space variables at the bottom and top of the $i$ th sub-section above ground respectively. Here $\mathbf{T}_{g i}=\exp \left(\mathbf{H}_{g i} \Delta x\right)$ and $\mathbf{A}_{g i}=\left(\mathbf{T}_{g i} \mathbf{H}_{g i}^{-1}-\mathbf{H}_{g i}^{-1}\right) \mathbf{B}_{g i}$

When $N_{1}$ is large enough, $N_{j}(z)$ and $k_{j}(z)$ also for the $j$ th subsection are assumed to be constant, and are calculated as: $N_{j}=N_{p}+f_{0} H_{0}+f(2 j-1) \Delta z / 2$ and $k_{j}=m\left(z_{0}+(2 j-1) \Delta z / 2\right)^{n}\left(j=1,2, \mathrm{~L}, N_{1}\right)$ respectively. And another set of state space equations for the $j$ th sub-section below ground are

$$
\frac{d}{d \bar{z}}\left\{\begin{array}{l}
u_{j}(\bar{z}) \\
M_{j}(\bar{z}) \\
Q_{j}(\bar{z}) \\
\varphi_{j}(\bar{z})
\end{array}\right\}=\left[\begin{array}{cccc}
0 & 0 & 0 & 1 \\
0 & 0 & 1 & -N_{j} \\
-k_{j} b & 0 & 0 & 0 \\
0 & 1 / E I & 0 & 0
\end{array}\right]\left\{\begin{array}{l}
u_{j}(\bar{z}) \\
M_{j}(\bar{z}) \\
Q_{j}(\bar{z}) \\
\varphi_{j}(\bar{z})
\end{array}\right\}{ }_{\left(j=1,2, \mathrm{~L}, N_{1}\right)}
$$

Where $u_{j}(\bar{z}), M_{j}(\bar{z}), Q_{j}(\bar{z})$ and $\phi_{j}(\bar{z})$ are the horizontal displacement, bending moment, shear force and rotation angle at a depth $\bar{z}$, respectively.

$$
\text { Let } \mathbf{H}_{j}=\left[\begin{array}{cccc}
0 & 0 & 0 & 1 \\
0 & 0 & 1 & -N_{j} \\
-k_{j} b & 0 & 0 & 0 \\
0 & 1 / E I & 0 & 0
\end{array}\right]
$$

In a similar way, we also can attain another set of relationship equations between two state space variables at the top and bottom of the $j$ th subsection below ground

$$
\mathbf{U}_{j}(\Delta z)=\mathbf{T}_{j} \mathbf{U}_{j}(0)\left(j=1,2, \mathrm{~L}, N_{1}\right)
$$

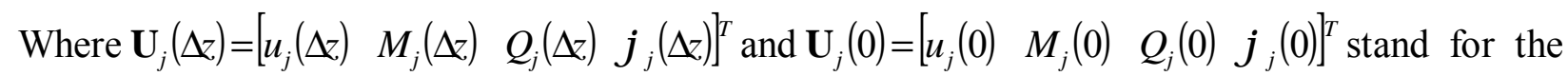
state space variables at the bottom and top of the $j$ th sub-section below ground respectively 
and $\mathbf{T}_{j}=\exp \left(\mathbf{H}_{j} \Delta z\right)$. From Eqs.(3-6),(8) and(10), a set of relationships between two state space variables at the pile head and base are obtained as:

$$
\begin{aligned}
& \mathbf{U}_{N 1}(\Delta z)=\mathbf{T}_{N 1} \mathrm{~L} \mathbf{T}_{j} \mathrm{~L} \mathbf{T}_{1} \mathbf{T}_{g N 0} \mathrm{~L} \mathbf{T}_{g i} \mathrm{~L} \mathbf{T}_{g 1} \mathbf{U}_{g 1}(0)+\mathbf{T}_{N 1} \mathrm{~L} \mathbf{T}_{j} \mathrm{~L} \mathbf{T}_{1}\left(\mathbf{T}_{g N 0} \mathrm{~L} \mathbf{T}_{g i} \mathrm{~L} \mathbf{T}_{g 2} \mathbf{A}_{g 1}\right. \\
& \left.+\mathbf{T}_{g N 0} \mathrm{~L} \mathbf{T}_{g i} \mathrm{~L} \mathbf{T}_{g 2} \mathbf{A}_{g 2}+\mathrm{L}+\mathbf{A}_{g N 0}\right)
\end{aligned}
$$

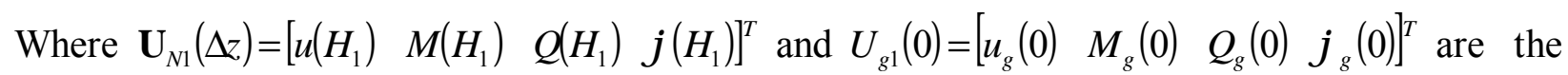
state space variables at the pile head and base respectively. Let $\mathbf{T}_{N 1} \mathrm{~L} \mathbf{T}_{j} \mathrm{~L} \mathbf{T}_{1} \mathbf{T}_{g N 0} \mathrm{~L} \mathbf{T}_{g i} \mathrm{~L} \mathbf{T}_{g 1}=\boldsymbol{\Omega}$ and $\mathbf{T}_{N 1} \mathrm{~L} \mathbf{T}_{j} \mathrm{~L} \mathbf{T}_{1}\left(\mathbf{T}_{g N 0} \mathrm{~L} \mathbf{T}_{g i} \mathrm{~L} \mathbf{T}_{g 2} \mathbf{A}_{g 1}+\mathbf{T}_{g N 0} \mathrm{~L} \mathbf{T}_{g i} \mathrm{~L} \mathbf{T}_{g 2} \mathbf{A}_{g 2}+\mathrm{L}+\mathbf{A}_{g N 0}\right)=\boldsymbol{\Gamma}$ Where $\boldsymbol{\Omega}$ is a fourth order matrix, $\boldsymbol{\Gamma}$ is a second order column vector. When both of the pile head and base are free, substitution of boundary conditions Eqs.(3) and (4) into Eq.(11) gives rise to two equations for finding $u_{g}(0)$ and $\varphi_{g}(0)$ at the pile head:

$$
\left\{\begin{array}{l}
u_{g}(0) \\
\varphi_{g}(0)
\end{array}\right\}=-\left[\begin{array}{ll}
\Omega_{11} & \Omega_{14} \\
\Omega_{41} & \Omega_{44}
\end{array}\right]^{-1}\left(\left[\begin{array}{ll}
\Omega_{12} & \Omega_{13} \\
\Omega_{42} & \Omega_{43}
\end{array}\right]\left\{\begin{array}{l}
M_{p} \\
Q_{p}
\end{array}\right\}+\left\{\begin{array}{l}
\Gamma_{1} \\
\Gamma_{4}
\end{array}\right\}\right)
$$

When the pile head is free while the pile base is fixed, substitution of Eqs.(3) and (5) into Eq.(11) yields two equations for finding $u_{g}(0)$ and $\varphi_{g}(0)$ at the pile head:

$$
\left\{\begin{array}{l}
u_{g}(0) \\
\varphi_{g}(0)
\end{array}\right\}=-\left[\begin{array}{ll}
\Omega_{21} & \Omega_{24} \\
\Omega_{31} & \Omega_{34}
\end{array}\right]^{-1}\left(\left[\begin{array}{ll}
\Omega_{22} & \Omega_{23} \\
\Omega_{32} & \Omega_{33}
\end{array}\right]\left\{\begin{array}{l}
M_{p} \\
Q_{p}
\end{array}\right\}+\left\{\begin{array}{l}
\Gamma_{2} \\
\Gamma_{3}
\end{array}\right\}\right)
$$

Hitherto, all the internal forces and deformations at the base of the $i$ th and $j$ th sub-sections can be calculated step by step by means of Eqs.(8) and (10) after $u_{g}(0)$ and $\varphi_{g}(0)$ are determined from Eq.(12) or Eq.(13). So far the above solution is called as a state space one.

\section{Validation}

A computation code for the present solution is programmed based on Matlab5.2's platform. Let us consider a reinforced-concrete and partially embedded pile as an numerical example in which calculated parameters are taken as: $H_{0}=15 \mathrm{~m}, H_{1}=30 \mathrm{~m}, b=1.8 \mathrm{~m}, E I=9.275 \times 106 \mathrm{kN} . \mathrm{m} 2$, $q(x)={ }_{0}, f_{0}=62.345 \mathrm{kN} / \mathrm{m}, Q_{p}=300 \mathrm{kN}, M_{P}=200 \mathrm{kN} . \mathrm{m}, N_{P}=10000 \mathrm{kN}, \quad z_{0}=0.4 \mathrm{~m}, n=0.5$ and $m=6 \times 103 \mathrm{kN} / \mathrm{m} 3.5$. We assume that both file head and base are free and the axial force reduces gradually up to zero from ground to the pile base in a linear fashion. Fig.(5) shows the distribution curves of horizontal displacement $u$ and bending moment $M$ along the pile axis by the state space solution (SSS) and the semi-analytical one (SAS) (Zhang L. et.al.,2011) respectively.

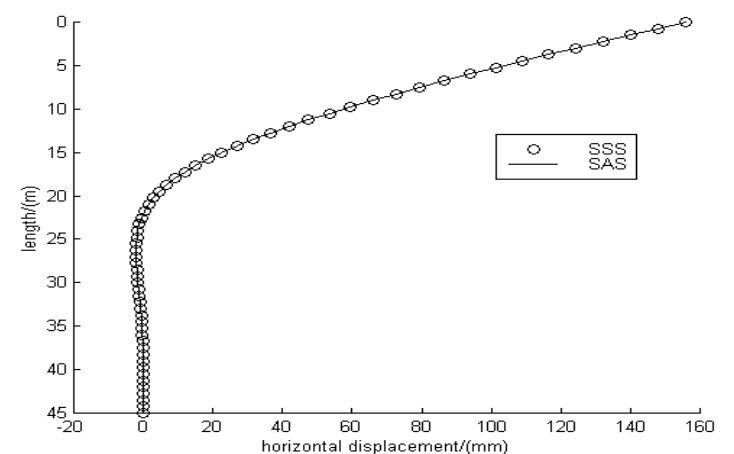

(a)

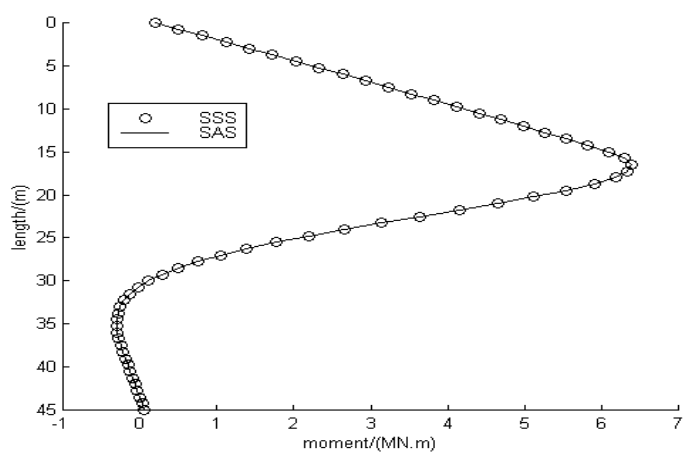

(b)

Figure.5 Bending responses, (a) horizontal displacement, (b) bending moment 
It can be seen from Fig.(5) that the horizontal displacement(or bending moment) curve calculated by SSS agrees well with that by SAS. Thus the present solution and its computation code are much reliable.

The relationships between maximum horizontal displacements $u_{m z x}$ versus sub-section number $\left(N_{0}+N_{1}\right)$ and ones between maximum moments $M_{\max }$ versus sub-section number $\left(N_{0}+N_{1}\right)$ are listed in Table.(1), where other parameters and applied loads are the same as those in the above example. It can be noted that both $u_{\max }$ and $M_{\text {max }}$ go on increasing with increasing sub-section number, their growth ratios sharply trend to zero. Therefore their convergence velocity is very fast. And Table.(1) also shows that they basically do no longer increase after $N_{0} \geq 80$ while $N_{1} \geq 160$.

Table.1 Relationships between maximum displacement,maximum moment and subsection number

\begin{tabular}{llllll}
\hline$N_{0}, N_{1}$ & $u_{\max }$ & $M_{\max }$ & $N_{0}, N_{1}$ & $u_{\max }$ & $M_{\max }$ \\
& $\mathrm{mm}$ & MN.m & & $\operatorname{mm}$ & MN.m \\
\hline 10,20 & 154.67 & 6.3528 & 40,80 & 156.28 & 6.3968 \\
15,30 & 155.58 & 6.3611 & 80,160 & 156.37 & 6.4015 \\
20,40 & 155.93 & 6.3870 & 160,320 & 156.40 & 6.4022 \\
\hline
\end{tabular}

\section{Conclusions}

The state space equations above and below ground for a partially embedded pile subjected to oblique loads are presented according to Euler-Bernoulli's and Winkler's linear elastic foundation beam theory respectively, in which the coefficient of sub-grade reaction utilizes a three parameters model. The formulations of internal forces and deformations at a depth for all the sub-sections below and above ground are deduced by means of matrix theory, boundary conditions and continuity-smooth conditions at the interface between two adjacent sub-sections. The computational code for the present solution is programmed by Matlab5.2. Compared with existing semi-analytical solution, the present one has higher convergence and accuracy, and is easy-programmed to be popularized.

\section{References}

[1] Karthigeyan, S., Ramakrishna,V.V.G.S.T and Rajagopal,K: submitted to Computers and Geotechnics (2006)

[2] Karthigeyan, S., Ramakrishna, V.V.G.S.T and Rajagopal,K. submitted to Journal of Geotechnical and Geo-environmental Engineering,ASCE (2007)

[3] Zheng, G. and Wang, L:submitted to Rock and Soil Mechanics(2009)

[4] Zhao, M.H: submitted to Journal of Hunan University (1987)

[5] Wang,Z.and Gong, X.N:submitted to Journal of University of Science and Technology of Suzhou (Engineering and Technology)(2005)

[6] Han,J.and Frost,J.D:submitted to International Journal of Numerical and Analytical Methods in Geo-mechanics(2000).

[7] Poulos, H.G. and Davis, E.H, Pile Foundation Analysis and Design, Wiley, New York,1980.

[8] Wu,H.L,Thrust Calculation of a Pile in the Composite Stiffness Principle and the Method of Double Parameters, China Communications Press, Beijing,2000.

[9] Wang,B.H.,Shang,G.X.,China's New Development of Bored Piles, China Communications Press, Beijing, 1999.

[10] Matlock,H., Reese,L.C:submitted to Journal of the Soil Mechanics and Foundation division, $\operatorname{ASCE}(1960)$.

[11] Zhang,L.,Gong,X.N.,Yu,J.L:submitted to Journal of Sichun University (Engineering Science Edition) (2011). 